Cipango $\begin{aligned} & \text { Cipango } \\ & \text { Cahiers d'études japonaises }\end{aligned}$

Hors-série | 2008

Autour du Genji monogatari

\title{
Le palais de Heian
}

Sur les pas du Genji

Following Genji's Footsteps - the Heian palace

\section{Marie Maurin}

\section{CpenEdition}

Journals

Édition électronique

URL : https://journals.openedition.org/cipango/601

DOI : $10.4000 /$ cipango.601

ISSN : 2260-7706

Éditeur

INALCO

Édition imprimée

Date de publication : 1 janvier 2008

Pagination : 185-213

ISBN : 978-2-85831-170-5

ISSN : 1164-5857

Référence électronique

Marie Maurin, «Le palais de Heian », Cipango [En ligne], Hors-série | 2008, mis en ligne le 27 février 2012, consulté le 30 juin 2021. URL : http://journals.openedition.org/cipango/601 ; DOI : https:// doi.org/10.4000/cipango.601

Ce document a été généré automatiquement le 30 juin 2021.

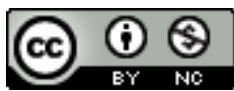

Cipango est mis à disposition selon les termes de la Licence Creative Commons Attribution - Pas d'Utilisation Commerciale 4.0 International. 


\title{
Le palais de Heian
}

\author{
Sur les pas du Genji \\ Following Genji's Footsteps - the Heian palace
}

Marie Maurin

1 Kiritsubo, le titre du premier chapitre du Roman du Genji, correspond à un pavillon du palais impérial (daidairi 大内裏) de Heiankyō 平安京, la dernière capitale du Japon de l'époque ancienne, fondée en 794 . Vers la fin du chapitre, Murasaki Shikibu mentionne ce même édifice, où réside la favorite de l'empereur, sous son nom chinois Shigeisha 淑 景舎, c'est-à-dire «clos du Paysage frais comme le printemps ». Mais elle donne fort peu de détails sur l'emplacement exact et l'aspect de cette partie du palais, comme c'est d'ailleurs le cas pour la plupart des lieux auxquels elle fait allusion dans son œuvre. Murasaki Shikibu n'a dû effectuer, selon toute vraisemblance, que des séjours très limités dans le temps et dans l'espace à l'intérieur du daidairi, ce qui pourrait expliquer en partie son laconisme. Quoi qu'il en soit, pour avoir une idée plus précise du cadre dans lequel évoluent les héros du Roman $d u$ Genji, il faut faire appel à d'autres sources d'information.

2 Les recherches archéologiques n'ont guère progressé, le site de Heiankyō ayant disparu très tôt dans le tissu urbain d'une autre ville, celle de Kyōto. Les fouilles ont permis d'exhumer quelques vestiges de la cour de l'Abondance et des plaisirs, Burakuin 豊楽 院, une vaste enceinte cérémonielle qui reposait en partie sur un soubassement maçonné. Mais les résultats restent très limités, d'autant que, comme on le verra plus loin, certains édifices du palais impérial consistaient en une ossature en bois surmontée d'un toit de bardeaux de cyprès dont le temps n'a guère eu de mal à effacer les traces. Outre les multiples sinistres dont elle a été victime durant toute la période de Heian, Heiankyō a été dévastée coup sur coup par deux gigantesques incendies vers la fin de l'époque ancienne ${ }^{1}$. Le premier s'est produit en 1177 (Angen 3) et en l'espace d'une nuit, il a anéanti quelque cent quatre-vingts quartiers dans la section de l'Est de la ville, entre la Cinquième avenue et l'avenue Konoemikado 近衛御門 (pl. 1 et 2). Plusieurs ensembles architecturaux et pavillons parmi les plus prestigieux et les plus imposants du palais impérial, dont la cour des Huit départements, Hasshōin 八省院, appelée aussi Chōdōin 朝堂院, et le pavillon de l'Ultime suprême, Daigokuden 大極殿, ont disparu 
dans les flammes, en même temps que la résidence du grand chancelier Fujiwara no Motofusa 藤原基房 (1145-1230), celles d'une dizaine de très grands personnages et une vingtaine de quartiers d'habitation ainsi que leur contenu (pl. 3 et 4). Un an plus tard (1178) presque jour pour jour, un second sinistre de grande ampleur a ravagé le sud de la section de l'Est et réduit en cendres une cinquantaine de quartiers ${ }^{2}$. La capitale n'était plus que l'ombre d'elle-même. C'est alors que le nom de Heiankyō (littéralement, la " capitale de la paix ») est tombé en désuétude et qu'il a été remplacé par celui de Kyōto. À partir du milieu du XIII ${ }^{\mathrm{e}}$ siècle, on a d'ailleurs désigné le terrain à l'abandon où s'élevait autrefois le palais impérial sous le nom de « lande du palais »uchino 内野.

Plan de la ville de Heian en français

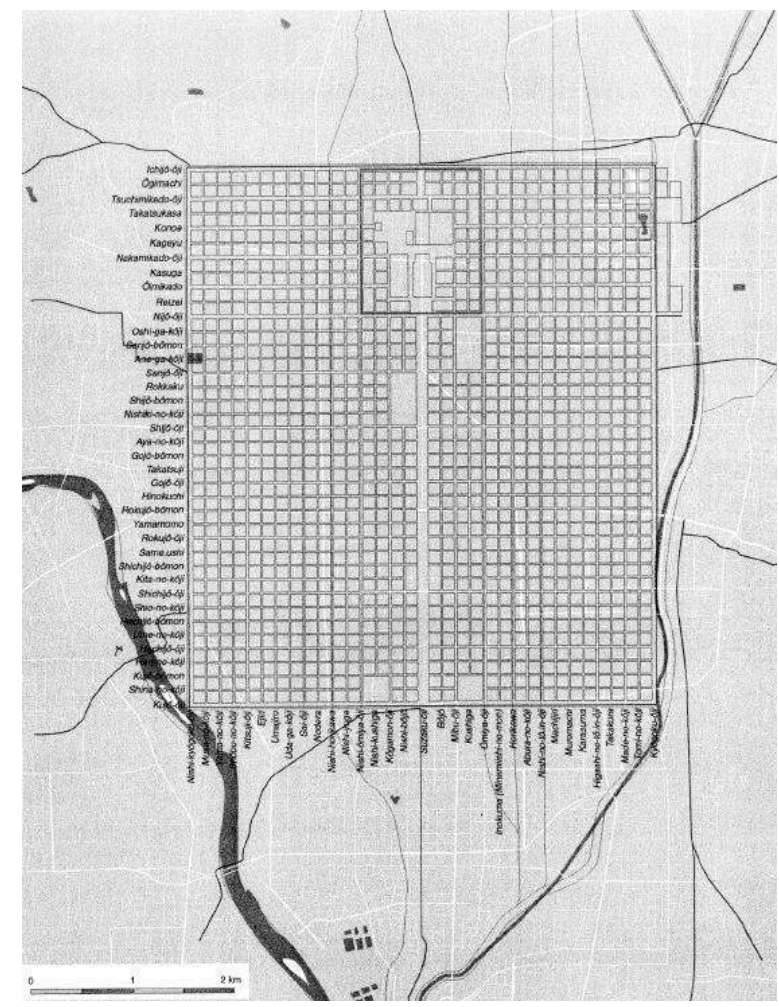

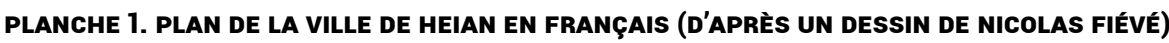

Plan de la ville de Heian en japonais 
平安京図

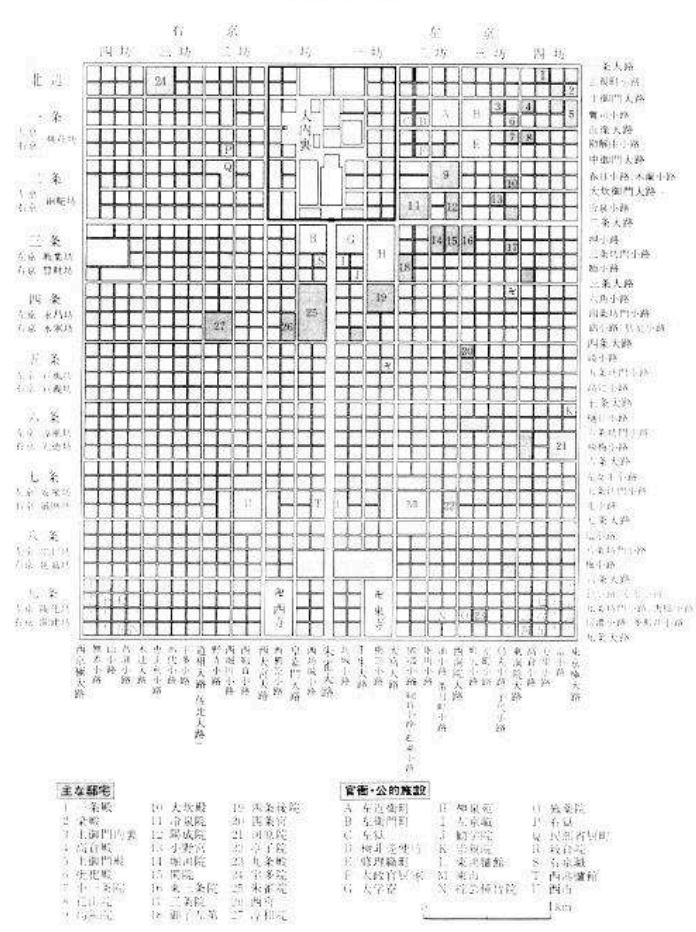

PLANCHe 2. PLAN de LA VILLE de heIAN EN JAPONAIS (D'APRÈs NAgAHARA KEIJI ET AL., Éd., NIHONSHI JITEN, IWANAMI SHOTEN, 1999, P. 1403)

Plan du palais impérial daidairi en français 


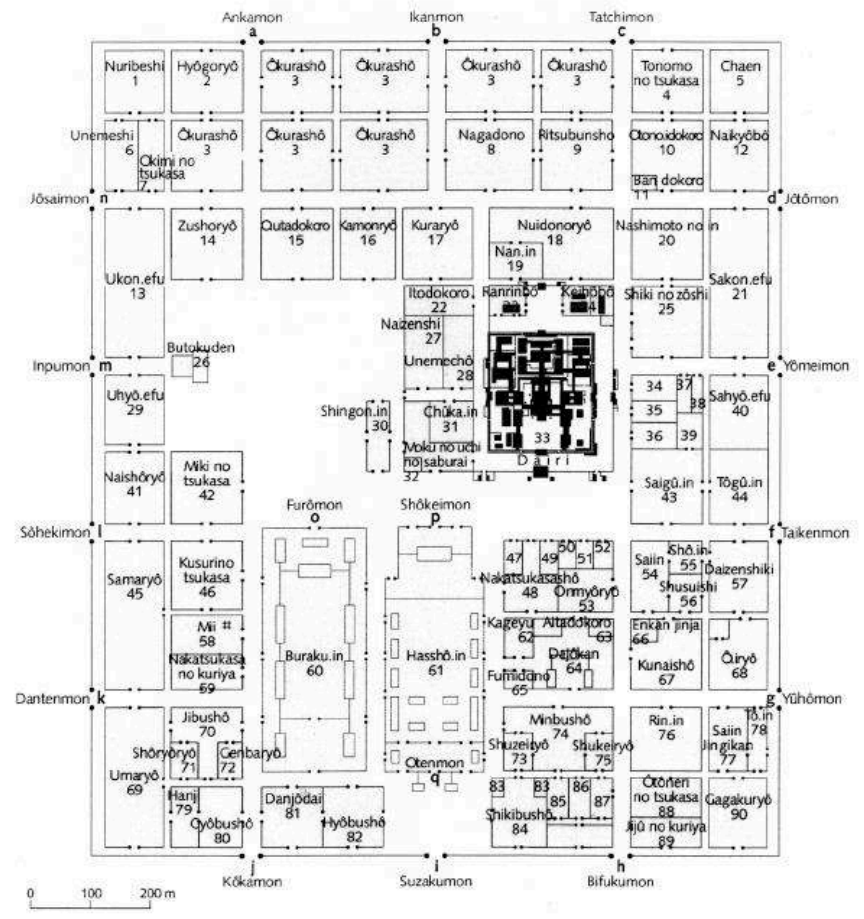

Le plan du palais impérial

PLANCHE 3. PLAN dU PALAIS IMPÉRIAL dAIDAIRI EN FRANÇAIS (D'APRÈS NICOLAS FiÉVÉ, ATLAS HISTORIQUE DE KYŌTO, UNESCO, 2008)
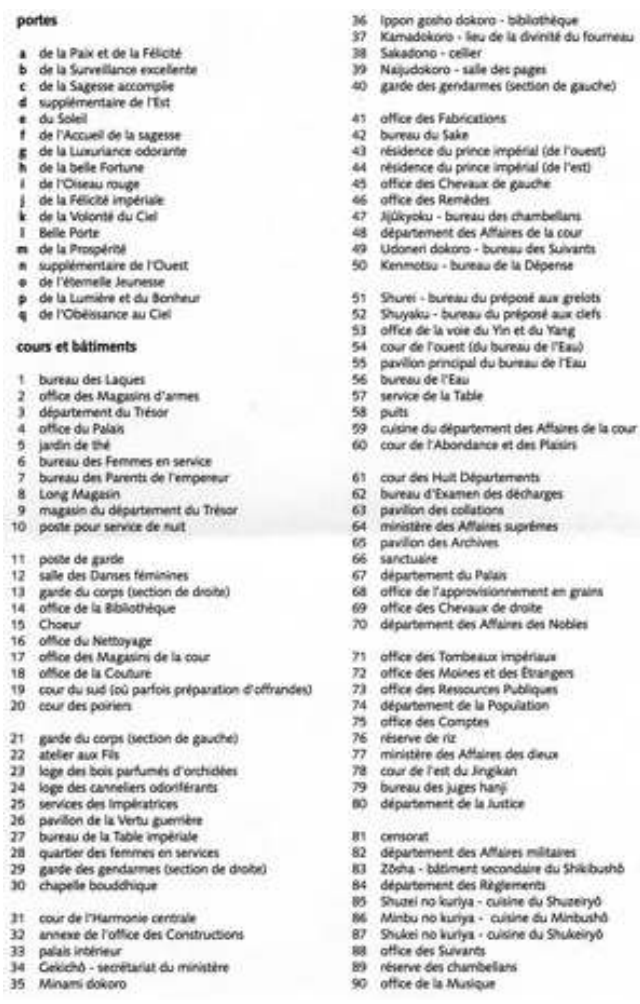


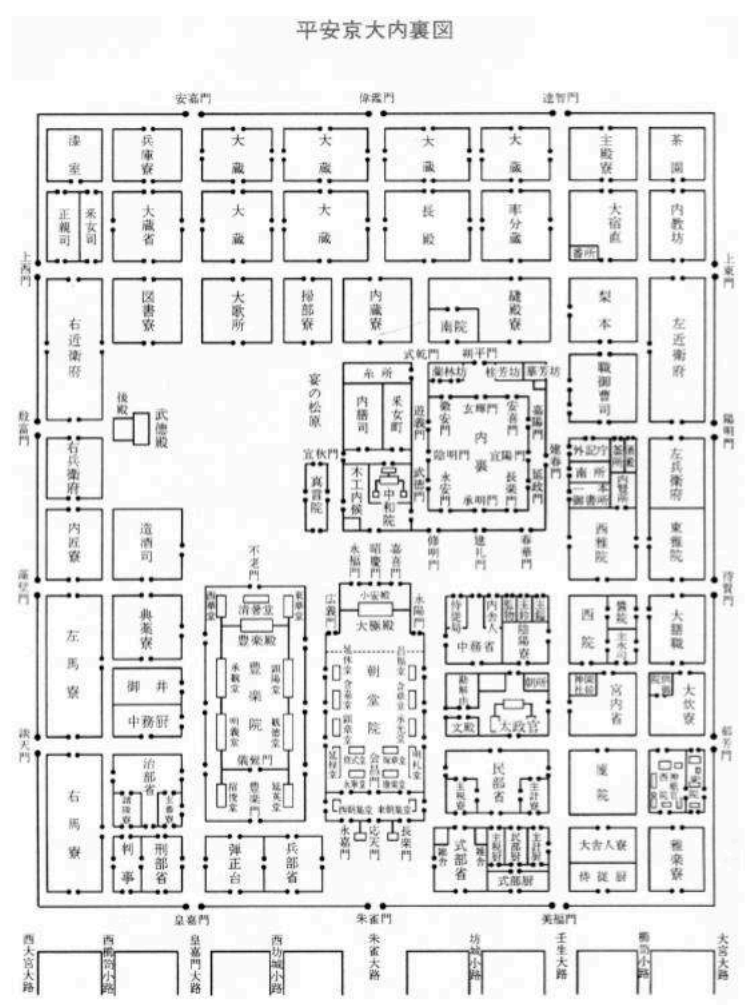

PLANCHE 4. PLAN du PALAIS IMPÉRIAL dAIDAIRI EN JAPONAIS (D'APRÈs NAgAHARA KEIJI ET AL., Éd., NIHONSHI JITEN, IWANAMI SHOTEN, 1999, P. 1404)

Les sources écrites et iconographiques sont heureusement plus éloquentes. Il existe en particulier plusieurs ensembles de plans de la capitale dont certains ont été réalisés dès l'époque de Heian pour garder trace des dimensions et de la configuration du palais de Heian $^{3}$. Le plus ancien de ces documents est le Kyūjozzu 宮城図 (Plan du palais impérial, pl. 9), dont on a conservé une copie datée de 1319 (Gen.ō 1). Celle-ci se présente sous la forme d'un rouleau où sont reproduits à l'encre rehaussée de couleurs légères des plans du palais impérial daidairi, de la résidence impériale dairi 内裏, de la cour de l'Harmonie centrale, Chūkain ou Chūwain 中和院, de la cour des Huit départements, Hasshōin, et de la cour de l'Abondance et des plaisirs, Burakuin 豊楽院. On y trouve par ailleurs mentionnés le nom des provinces qui ont effectué des travaux sur telle ou telle partie du palais ainsi que les incendies survenus dans la capitale entre 960 (Tentoku 4) et 1080 (Eihō 2), ce qui donne à penser que les plans du Kyūjōzu ont dû être réalisés dès cette période ${ }^{4}$. Le livre XLII du Engishiki 延喜式 (Règlements de l'ère Engi) contient lui aussi des plans du palais (Kyūzu 宮図), dont une copie du XIV ${ }^{\mathrm{e}}$ siècle qui fait partie des collections du Musée national de Kyōto ${ }^{5}$. Enfin Uramatsu Mitsuyo 裏松光世 (1736-1804), un noble de cour de l'époque d'Edo, a compilé une œuvre monumentale en trente-deux volumes, intitulée Daidairizu kōshō 大内裏図考證 (Recherches sur le plan du palais), où l'on trouve une description détaillée non seulement de chaque bâtiment du palais, mais aussi du mobilier qu'il contenait et même des végétaux qui poussaient dans les cours ${ }^{6}$. Les indications données par ces trois ensembles de plans sont par 
ailleurs confirmées par les représentations de diverses parties du palais qui figurent sur certains rouleaux de peinture, en particulier le Nenjūgyōji emaki 年中行事絵巻 (Rouleau enluminé du cycle des célébrations de la cour) ${ }^{7}$.

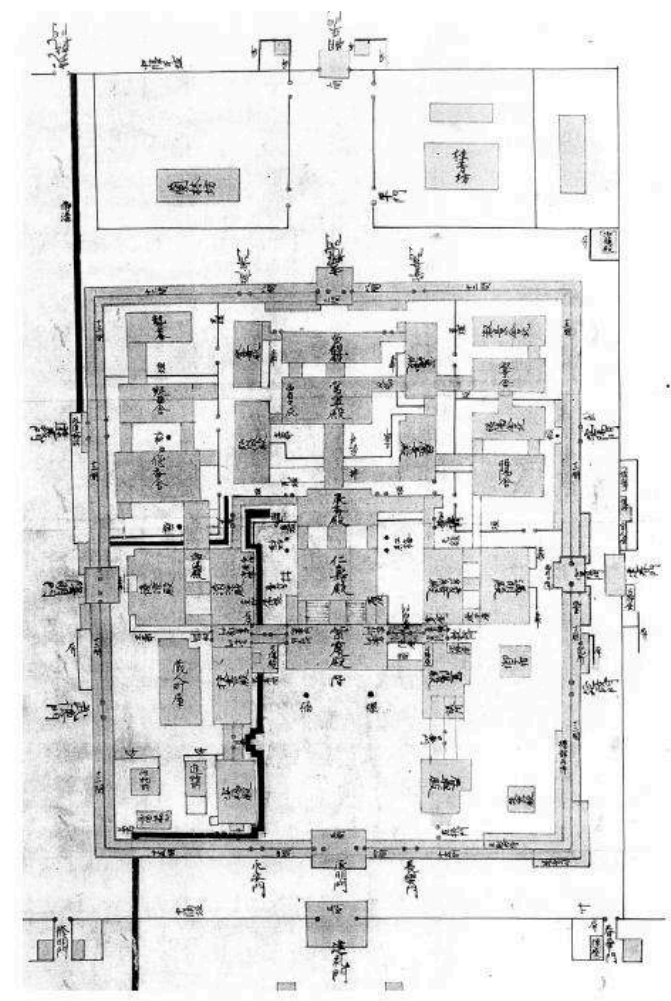

PLANCHe 9. PLAN de LA RÉSIDENCE IMPÉRIALE, MANUSCRIT, COPIE de 1319,KYŪJō ZU, YōMeI SŌSHO : KIROKU MONJO, BESSHŪ, SHIBUNKAKU SHUPPAN, 1996

\section{Heiankyō, une ville à l'image de la capitale des Tang}

Comme ceux des capitales précédentes du Japon et de la Chine, les bâtisseurs de la ville de Heian ont soigneusement choisi l'emplacement de la résidence de l'empereur et du siège de l'administration centrale en fonction non seulement de la topographie mais aussi de la géomancie. Dans la cosmogonie chinoise, la capitale est en effet considérée comme le centre de convergence des influences cosmiques à partir duquel le souverain fait régner l'harmonie sur l'empire. Les spécialistes de la géomancie se sont assurés que la nature du terrain et sa configuration, et en particulier celle des montagnes et des cours d'eau étaient conformes à des impératifs bien définis. Les montagnes étant censées être imprégnées d'énergie vitale, une des tâches des géomanciens consistait à repérer celles qui étaient particulièrement chargées de forces bénéfiques, à conduire ces forces vers le bas et, partant, à les guider vers la capitale par le biais des cours d'eau soigneusement canalisés. Ils ont ainsi créé un site favorable qui bénéficiait d'influences fastes, tout en étant protégé de celles qui pouvaient être néfastes ${ }^{8}$. Et comme on le 
verra par la suite, l'organisation spatiale des cours (in 院) et des édifices de Heiankyō suivait les mêmes règles.

Les Japonais ont en outre emprunté à l'architecture chinoise deux autres grandes constantes, à savoir le principe d'axialité et la cour carrée fermée sur ses quatre côtés, qui donne aux ensembles architecturaux chinois et japonais l'aspect d'une succession de mondes clos plus ou moins vastes en fonction de leur importance ${ }^{9}$. Ils n'ont cependant pas doté Heiankyō des murailles protectrices qui entouraient Chang'an 長 安, la capitale des Tang (618-907), et la quasi-totalité des cités chinoises dignes de ce nom. Les remparts de la capitale se sont limités à une porte imposante au nom éloquent, Rashōmon 羅城門, ou porte de l'Enceinte, située au sud, à l'entrée principale de la ville, et rappelant à bien des égards celle de Chang'an ${ }^{10}$. À l'instar de la capitale des Tang, Heiankyō avait un tracé en damier rectangulaire établi à partir d'un axe principal nord-sud, l'avenue de l'Oiseau rouge, Suzaku ooji 朱雀大路, et constitué par un réseau d'artères - d'une longueur totale de 323 kilomètres - orientées les unes nordsud, les autres est-ouest et se coupant à angle droit. La ville avait des proportions plus modestes - 5,312 kilomètres $\left(1508 j \bar{o}^{11}\right)$ du nord au sud et 4,569 kilomètres $(1753 j \bar{o})$ d'est en ouest - que celles de la capitale des Tang, qui formait un gigantesque quadrilatère de 9,5 kilomètres d'est en ouest sur 8,2 kilomètres du nord au sud.

\section{Le palais impérial daidairi, une juxtaposition d'enclos de pisé}

6 À l'intérieur du quadrilatère qui marquait les limites de Heiankyō, le palais daidairi ${ }^{12}$ dessinait un rectangle de dimensions plus réduites, mais de mêmes proportions, qui était lui aussi situé de part et d'autre d'un axe de symétrie nord-sud et se trouvait adossé au côté nord de la ville. De même que le palais ou plutôt les palais de Chang'an à l'époque des Tang - le Taijigong 太极宮, palais du Faîte suprême, auquel est venu s'ajouter en 662 un second palais, le Daminggong 大明宮, palais de la Grande clarté, situé au nord-est de l'enceinte proprement dite de la capitale des Tang - et celui de Nara, le palais de Heian ne se trouvait pas au centre de l'agglomération ainsi qu'il était spécifié dans la définition de la capitale idéale, image de l'Univers donnée par le Zhouli 周礼 (Rituel des Zhou, IV ${ }^{\mathrm{e}}-\mathrm{III}{ }^{\mathrm{e}}$ siècles av. J.-C.). Pourtant, les maîtres du Japon n'auraient pas dû suivre les architectes du continent sur ce point puisque les considérations qui ont poussé ces derniers à transférer la cité interdite chinoise au nord de Chang'an étaient d'ordre, non pas cosmologique, mais essentiellement pratique. En effet, le nord de la capitale était plus élevé que son centre, si bien qu'en été la chaleur y était un peu moins accablante. Dans le même temps, les deux marchés qui se trouvaient jusque-là au nord de Chang'an ont été déplacés vers le sud et implantés à proximité de l'axe estouest très fréquenté par où transitaient les marchandises et qui divisait la ville en deux parties bien distinctes ${ }^{13}$. Il faut croire qu'en bâtissant une capitale calquée sur celle de la Chine, les maîtres de l'archipel entendaient affirmer, à l'intérieur comme à l'extérieur du pays, le pouvoir de la cour et de l'administration impériale.

7 Le palais impérial de Heiankyō mesurait 1373 mètres (460 jō) du nord au sud sur 1146 mètres $(384 j \bar{j})$ d'est en ouest et il couvrait une superficie de 163,5382 hectares équivalant à celle de quatre-vingts quartiers chō 町, le chō constituant l'unité de base de la structure de la capitale. Le quadrilatère ainsi formé était matérialisé par une haute enceinte de pisé qui jouxtait le côté nord de la ville. Le palais donnait au sud sur la Deuxième Avenue, Nijōoji 二条大路, à l'ouest sur l'avenue Occidentale du Palais, Nishi 
no Ōmiya ōji 西大宮大路, et à l'est sur l'avenue du Palais, Ōmiya ōji 大宮大路 (pl. 1 et 2). Le mur d'enceinte du daidairi était percé de quatorze portes dont trois s'ouvraient au sud, trois au nord, quatre à l'est et à quatre à l'ouest. La plus monumentale d'entre elles, celle de l'Oiseau rouge, Suzakumon 朱雀門, se trouvait dans l'axe de l'avenue du même nom qui constituait l'artère principale de la capitale et aboutissait à la porte de l'Enceinte, Rashōmon, tout au sud de la ville. Chacune des portes du palais était surmontée d'un écriteau en bois où était calligraphié son nom. La plupart reposaient sur un soubassement agrémenté de marches de pierre interdisant le passage à tout véhicule tiré par des bœufs. Certaines faisaient toutefois exception à la règle, entre autres la porte de la Luxuriance odorante, Ikuhōmon 邰芳門, la porte supplémentaire de l'Est, Jōtōmon 上東門, et la porte de l'Accueil des Sages, Taikenmon 待賢門, à l'est, et la porte de la Volonté du Ciel, Dantenmon 談天門, à l'ouest, par où passaient les convois chargés de matériaux ou de provisions ainsi que les voitures de certains personnages de haut rang, princes impériaux, régent et autres ministres qui jouissaient du privilège de pénétrer dans le palais impérial autrement qu'à pied. Une des scènes du rouleau de peinture Shigisan engi emaki 信貴山縁起絵巻 (Rouleau enluminé des légendes du mont Shigi, c. 1155-1180) donne une idée de l'aspect que devaient avoir les portes du palais de Heian ${ }^{14}$.

8 Le palais impérial abritait trois ensembles architecturaux de proportions imposantes qui étaient tous protégés par un mur de pisé. Chacune de ces trois cours (in) ou enceintes principales renfermait quantité de pavillons qui étaient reliés entre eux par des passages et formaient avec eux des cours intérieures plus ou moins vastes (pl. 3 et 4). Deux d'entre elles se trouvaient au sud du palais impérial, tandis que la troisième occupait le centre du daidairi. Ces trois enceintes monumentales étaient elles-mêmes entourées de tous côtés par un très grand nombre de bâtiments, pour la plupart des bureaux de l'administration, qui se trouvaient, eux aussi presque toujours à l'intérieur d'un enclos de pisé percé de portes. D'une manière générale, les pavillons et les édifices que contenait chaque enceinte étaient reliés à la fois par des couloirs protégés par un toit ( $r \bar{o}$ 廊; watarō 渡廊) et par des passages couverts (watadono 渡殿). Ce réseau d'édifices et de passages délimitait de petites cours rectangulaires couvertes de sable ou de gravier et parfois agrémentées de végétaux. Les passages couverts et les couloirs étaient assez spacieux pour qu'on puisse les reconvertir en pièces, si besoin était. Dans ses notes journalières (Shunki 春記), Fujiwara no Sukefusa 藤原資房 (1007-1057) rapporte qu'en 1040 (Chōkyū, 1.10.22.), la résidence de Fujiwara no Norimichi 藤原教通 (996-1057) a fait provisoirement office de palais impérial et il explique en détail comment les passages ont été transformés en bureaux et autres locaux réservés à l'usage du souverain ${ }^{15}$.

9 L'une des trois enceintes monumentales du daidairi abritait la résidence impériale (dairi, littéralement "palais intérieur ») dans lequel le souverain passait le plus clair de son temps. Les deux autres, qui avaient pour nom enceinte des Huit Départements, Hasshōin, et enceinte de l'Abondance et des Plaisirs, Burakuin, jouaient un rôle capital à l'occasion des grands rassemblements de la cour ${ }^{16}$. Le bâtiment principal de la cour des Huit Départements, appelé pavillon de l'Ultime Suprême, Daigokuden, contenait le takamikura 高御座, lieu privilégié entre tous situé dans l'axe de l'avenue de l'Oiseau rouge Suzaku oji depuis lequel l'empereur était censé contribuer au règne de l'harmonie dans le pays en lui dispensant ses incommensurables bienfaits. C'est dans ce 
cadre très structuré et hautement hiérarchisé que s'est déroulée la quasi-totalité des activités de l'empereur et de la cour durant l'essentiel de l'époque de Heian.

Dans la partie est du palais de Heian, il y avait par ailleurs deux enceintes de dimensions plus modestes, appelées enceinte de l'Est, Higashi no gain 東雅院 ou Tōzenbō 東前坊, et enceinte de l'Ouest, Nishi no gain 西雅院 ou Saizenbō 西前坊, qui constituaient à elles deux la maison du prince héritier ${ }^{17}$. La résidence proprement dite se trouvait dans le Nishi no gain, alors que le Higashi no gain était réservé aux banquets et autres divertissements. L'emplacement de la résidence du prince héritier de Heiankyō était conforme aux prescriptions du Yijing 易経 (Livre des mutations), d'après lequel la direction de l'est correspond à "ce qui vient après " et, par voie de conséquence, à « celui qui succède [à son père] », le fils aîné, en l'occurrence le prince héritierM VB2012-02-27T18:53:00. L'agencement de chacune des deux parties du Tōgūbō rappelait quelque peu celui de la résidence impériale avec une porte principale au sud donnant sur une vaste cour dominée par un grand pavillon à usage cérémoniel. Si l'on en croit le Nihon Montoku tennō jitsuroku 日本文徳天皇実録 (Chroniques véridiques du règne de l'empereur Montoku), à partir de l'année 850 , on a désigné la résidence du prince héritier sous le nom de Tōgūbō 東宮坊 (var. 春宮坊) ${ }^{18}$. Mais il semble que l'endroit n'ait plus servi de résidence à l'héritier du trône après le décès, survenu en 923 au Tōgūbō, du prince héritier Yasuaki shinnō 保明親王 (903-923), le deuxième fils de l'empereur Daigo 醍醐天皇. D'après le Shoyuki 小右記 (Notes journalières du ministre de droite d'Ononomiya), il aurait pris dès lors le nom de Kotōgū 古東宮 (littéralement "Ancienne résidence du prince héritier») ${ }^{19}$. Il semble donc qu'au moment où Murasaki Shikibu a écrit le Roman du Genji, le prince héritier n'occupait plus l'ensemble architectural que les bâtisseurs de Heiankyō avait édifié à son intention.

11 En dehors des nombreuses cours intérieures plus ou moins grandes dont il était constitué, le daidairi ne comportait qu'un seul espace ouvert dépourvu de construction, une vaste esplanade située à l'ouest de la résidence impériale à proximité du pavillon de la Vertu guerrière, Butokuden 武徳殿, où le souverain assistait au tir à l'arc monté et aux courses de chevaux, le $5^{\mathrm{e}}$ jour de la $5^{\mathrm{e}}$ lune ${ }^{20}$. La structure assez dense constituée par une juxtaposition d'enclos protégés par des murs de pisé qui était celle du daidairi contribuait à lui donner une configuration pour le moins singulière. À l'instar de la ville dans laquelle il s'inscrivait, le palais de Heian avait en effet l'aspect d'un monde clos tourné vers l'intérieur et rigoureusement compartimenté.

\section{Architecture continentale et architecture vernaculaire}

12 Si l'organisation spatiale de la capitale et du palais de Heian était dans l'ensemble très proche de celle de Chang'an, les pavillons du palais n'étaient pas tous conformes au modèle continental. En fait, deux types d'architecture coexistaient dans le daidairi avec, d'une part, des pavillons qui s'inspiraient directement de ceux des ensembles palatiaux de la Chine, à l'instar des établissements bouddhiques, et, de l'autre, une architecture de type domestique. Dans le Shinsarugakuki 新猿楽記 (Notes sur les nouveaux divertissements), Fujiwara no Akihira 藤原明衡 (989-1066) donne un aperçu des différents métiers exercés par les habitants de la capitale. Il dresse ainsi le portrait de l'époux de la huitième fille de la famille d'Uemon no jō 右衛門尉, un architecte du cinquième rang qui excellait dans les deux styles d'architecture. D'après Fujiwara no Akihira, ce personnage connaissait le plan, les proportions et les différents éléments 
non seulement des édifices des deux grandes enceintes cérémonielles du palais (Hasshōin et Burakuin) et des diverses sortes de pavillons des établissements bouddhiques, mais aussi des constructions de style japonais ${ }^{21}$.

Comme ceux des monastères bouddhiques, les grands pavillons de style chinois du palais de Heian reposaient sur un soubassement qui consistait en général en un remblai de terre recouvert d'un appareillage de pierres ou de tuiles plates auquel on accédait par des escaliers aménagés sur ses quatre faces. Sur la terrasse ainsi formée se dressait l'ossature en bois de l'édifice dont la partie centrale (moya 母)屋) était constituée par de robustes piliers reposant chacun sur un socle de pierre. Les parties visibles de la structure en bois étaient peintes en rouge, et l'édifice était couronné par une imposante toiture recouverte de tuiles vernissées de couleur verdâtre ou bleuâtre dont le bord incurvé à la manière des toitures chinoises débordait largement de la charpente centrale. Le moya des pavillons était souvent prolongé sur ses quatre côtés par une galerie (hisashi 庇) et les bâtiments les plus importants comportaient une galerie supplémentaire (mago hisashi 孫庇 ou mata hisashi 又庇).

En revanche, les bâtiments de la résidence impériale dairi contrastaient vivement avec les édifices officiels du daidairi dans la mesure où ils n'avaient pas de soubassement et étaient constitués d'une ossature en bois reposant sur des pilotis de bois et surmontée d'une toiture de bardeaux de cyprès hiwada buki 檜皮莫き, à l'exception du faitage qui était en tuiles ${ }^{22}$. Les rouleaux de peinture et en particulier le Nenjūgyōji emaki et le Ban dainagon emaki 伴大納言絵巻 (Rouleau enluminé du grand conseiller Tomo, XII ${ }^{e}$ siècle) donnent des exemples éloquents des deux types d'architecture qui coexistaient dans l'architecture palatiale de l'époque de Heian ${ }^{23}$. En ce qui concerne les dimensions des édifices de la capitale de Heian, on ne peut se fonder sur la valeur que prendra ultérieurement la travée ken 間, c'est-à-dire de l'intervalle entre deux piliers qui sert d'unité de référence pour la construction des bâtiments, dans l'architecture japonaise traditionnelle ${ }^{24}$. À l'instar de ce qui se passait dans les capitales chinoises, la longueur de l'unité de référence devait varier en fonction de l'importance de l'édifice ${ }^{25}$. On a tout lieu de supposer que les bâtisseurs japonais ont eu connaissance des traités d'architecture chinois, dont le plus ancien qui ait été conservé, le Yingzao fashi 菅造法 式 (Règlements et normes de la construction), a été présenté en 1100 à la cour des Song ${ }^{26}$.

\section{La résidence impériale, dairi}

La résidence impériale de Heian, où l'empereur passait le plus clair de son temps, se trouvait au nord des deux enceintes cérémonielles du Hasshōin et du Burakuin ${ }^{27}$. Le plan détaillé du dairi que contient le Kyūjōzu est particulièrement instructif à cet égard dans la mesure où il indique non seulement l'emplacement des pavillons, leur nom et les passages qui les reliaient, mais aussi le type de séparation - mur de pisé, haie, clôture... - et les portes qui structuraient l'espace intérieur de la résidence (pl. 9). Le Kyūjōzu va même jusqu'à mentionner les végétaux qu'on y trouvait, les puits et le tracé du petit canal (mikawamizu 御溝水) qui la traversait du côté de l'ouest. La résidence impériale dessinait un quadrilatère au centre du daidairi, comme à Nara, à ceci près qu'elle était plus étendue en direction de l'est. Elle était entourée par une double enceinte de pisé dont le mur extérieur (naka no e 中の重) mesurait 73 jō (env. $218 \mathrm{~m}$ ) d'est en ouest sur 100 jō (env. 298,5 m) du nord au sud. Les dimensions du mur intérieur 
(uchi no $e$ 内の重) étaient de 58 jō (env. $173 \mathrm{~m}$ ) d'est en ouest et de $72 \mathrm{jō}$ (env. $215 \mathrm{~m}$.) du nord au sud. À l'ouest et au nord, l'espace entre les deux enceintes était suffisant pour abriter plusieurs édifices. C'est ainsi qu'au sud-ouest, on trouvait la cour de l'Harmonie centrale, Chūkain, qui consistait en un enclos de pisé à l'intérieur duquel s'élevait le pavillon de la Félicité, Shinkaden 神嘉殿. Le souverain se rendait dans le Shinkaden trois fois par an, d'une part à la sixième et la onzième lune à l'occasion du Jinkonjiki 神 今食 où il partageait un repas rituel avec les divinités, et de l'autre à la onzième lune, lors de la célébration des Prémices annuelles Niinamesai 新嘗祭 ${ }^{28}$. Le Shinkaden était situé très précisément au centre du palais et dans l'axe de l'avenue de l'Oiseau rouge et du takamikura, le lieu où l'empereur siégeait dans le Daigokuden. Il semble donc que l'on puisse dire que la résidence impériale de Heian occupait le centre du palais comme dans les différentes capitales précédentes du Japon ancien. En remontant vers le nord, l'espace entre les deux enceintes du dairi était occupé par le quartier des femmes en service (Unemechō 采女町), le bureau de la Table impériale (Naizenshi 内膳司), et l'atelier aux Fils (Itodokoro 禾所), ainsi que par trois pavillons appelés loge des Bois parfumés (Ranrinbō 蘭林坊), loge des Canneliers odorants, Keihōbō 桂芳坊, et loge des Senteurs de fleurs, Kahōbō 華芳坊, où séjournaient, entre autres, les moines qui venaient au palais. Mais à l'est et au sud, les deux enceintes étaient très rapprochées et il n'y avait pas le moindre édifice entre elles (pl. 5 et 6).

Plan de la résidence impériale dairi en français

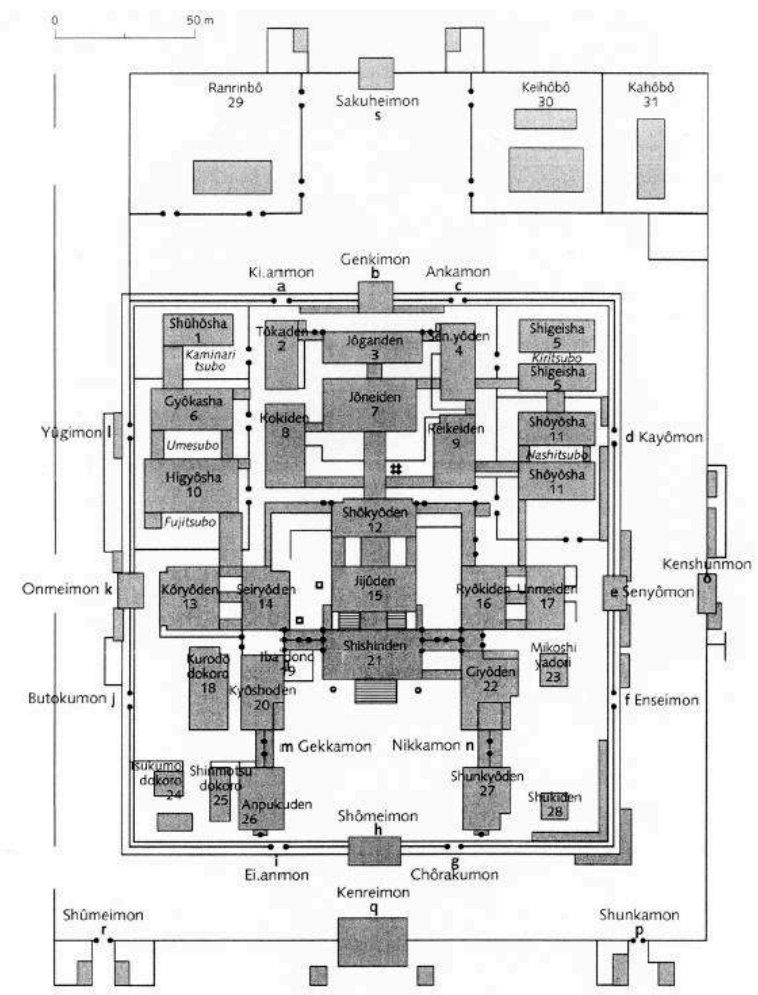

PLANCHE 5. PLAN de LA RÉSIDENCE IMPÉRIALE dAIRI EN FRANÇAIS (D'APRÈs NICOLAS FIÉVÉ, ATLAS HISTORIQUE DE KYŌTO, UNESCO, 2008) 
portes

a de 'agritable Tranquilite

c de la Tranquilltét et de la Fescicté

d de la felicicté et du principe Yó

ide 'omnipresent principe

du cos plasismement durables

$g$
$h$ des Plasirs durables
de la Clarte regue

de la Tranquilite etemelle

de la Vertu suerriere

de la Clurté du princlpe in
de l'Accomplissement et du Devir

$m$ de l'telat de la lune

- du vgoureux Paintemper

a des Ceremonies

$t$ de l'Ordire et de la Clarte

cours et bastiments

1 loge des Senteurs multiples
2 pavilon du Développerment de I'Edat

pavilion de la Vision correcte

pavilon de I'omnipresente Lumizere

loge du Paysage frabic comme le printemps
loge des Fleurs non zetives

palas de la Tranquilite hubiturete

pavilon de lExpansion du Beau

10 loge des Effluves parfumets

11 loge du rafieux prinope $Y$.

2 pavilion des Fragrances durabiles

3 pavilon arriere

pasillon de Purrete et de Fraicheur

16 pavillon des Brocarts

17 pavillon de la Chaleur et de la Clarte

18 Chancelierie perivé

11 pasvillon de er ftotole potaire

22 pavillon du benéfique principe $Y$

abri du palanquin imperial

6. pavillon de la Tranquillate et du Bonheur

27 pavillon des Plasisis du printemps

pavillon des Ustensiles rouges

loge des bis partumes d'orchides

31 loge des Sentevirs de Ferits

\section{PLANCHE 5 (SUITE). LÉgENDE}

Plan de la résidence impériale dairi en japonais

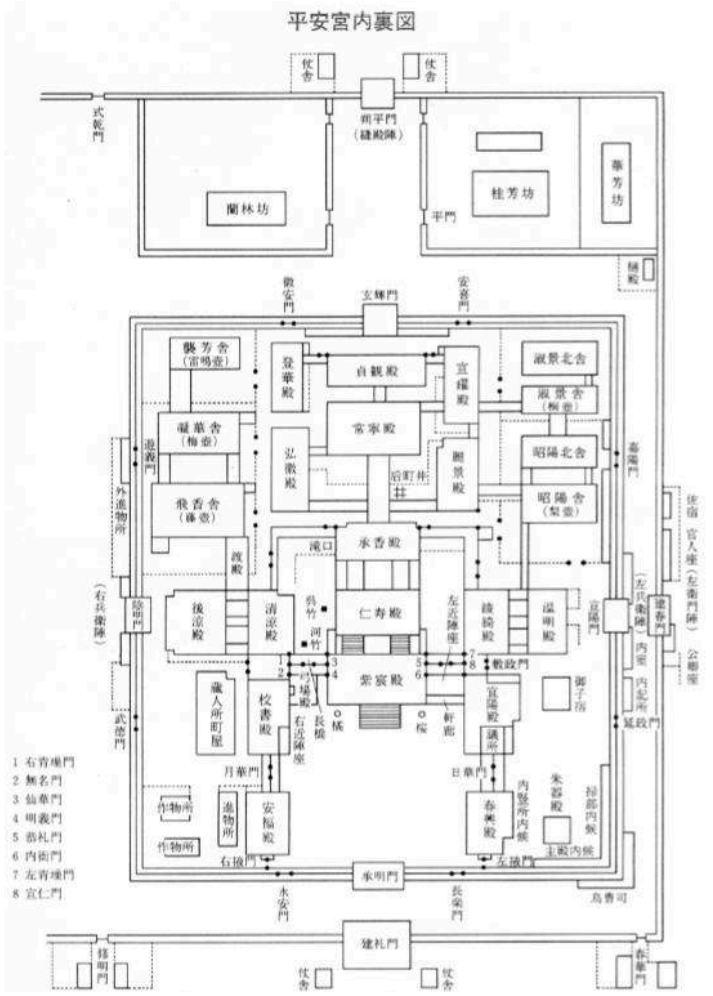


PLANCHE 6. PLAN de LA RÉSIDENCE IMPÉRIALE DAIRI EN JAPONAIS (D'APRÈs NAgAHARA KEIJI ET AL., ÉD., NIHONSHI JITEN, IWANAMI SHOTEN, 1999, P. 1405)

16 L'enceinte extérieure de la résidence impériale comportait huit portes dont quatre s'ouvraient au sud. L'accès principal de cette enceinte était la porte des Cérémonies bien organisées, Kenreimon 建礼門, qui comportait deux postes de garde jōsha 杖舎 placés légèrement en avant $\mathrm{du}$ mur de pisé et était encadrée par trois portes secondaires, la porte de l'Éclat printanier, Shunkamon 春華門, à l'est, et la porte de l'Ordre et de la Clarté, Shumeimon 修明門, et celle de l'Harmonie centrale Chūkamon 中和門, à l'ouest. Chacune des deux portes Shunkamon et Shumeimon était renforcée par deux maisons de gardes placées en avant de l'entrée. Le nord de l'enceinte extérieure du dairi était doté de deux portes, celle du Nord paisible, Sakuheimon 朔平 門, et celle des Rites perpétuels, Shikikenmon 式乾門, placée à l'ouest de la première. Les deux autres faces de l'enceinte extérieure n'avaient qu'une seule porte chacune, la porte du bel Automne, Gishūmon 宜秋門, à l'ouest, et la porte du vigoureux Printemps, Kenshunmon 建春門, à l'est, et ces deux accès étaient solidement protégés par d'importants corps de garde. L'enceinte intérieure du dairi comptait quant à elle douze portes, trois sur chacun de ses côtés. La plus importante s'ouvrait au sud et elle avait pour nom porte de la Clarté reçue, Shōmeimon 承明門. Le début du cinquième rouleau du Nenjūgyōji emaki, qui est consacré aux banquets, montre à quel point l'espace était réduit entre la porte de l'enceinte extérieure dite du vigoureux Printemps, Kenshunmon, et la porte de l'enceinte intérieure dite de l'Omniprésent principe yō (ch. yang), Sen.yōmon 宣陽門 ${ }^{29}$. La scène représentée donne une idée de la façon dont la résidence impériale était protégée. Pour y pénétrer, il fallait franchir plusieurs postes de garde qui disposaient de listes des personnes autorisées à y entrer, mais les vols n'en étaient pas moins très fréquents si l'on en juge par les documents de l'époque.

Le dairi proprement dit se composait de dix-sept pavillons (den 殿), cinq clos (sha 舎) et divers édifices secondaires qui étaient reliés entre eux par des passages plus ou moins larges. Comme dans la capitale chinoise, la partie sud de cet ensemble architectural était réservée aux activités officielles de l'empereur et la partie nord, appelée palais Arrière, $k \bar{o} k y \bar{u}$ 後宮, aux appartements privés du souverain ${ }^{30}$.

Shōmeimon, la porte principale du dairi, menait vers la partie de la résidence qui servait de cadre à la vie publique de l'empereur. Elle donnait sur une vaste cour, oniwa 大庭, où les hauts dignitaires et les fonctionnaires s'alignaient en bon ordre pour saluer le souverain. Le ōniwa était dominé par un imposant bâtiment de neuf travées d'est en ouest sur trois du nord au sud, le pavillon de l'Étoile polaire, Shishinden 紫宸殿, appelé aussi pavillon du Sud, Nanden 南殿 ou Naden 南殿, ou encore pavillon Avant, Zenden 前殿. Le Shishinden était l'édifice cérémoniel le plus important de la résidence impériale et, pour y accéder, il fallait gravir un escalier de dix-huit marches encadré à droite par un cerisier à fleur simple sakura et à gauche par un oranger tachibana (citrus tachibana C. Tanaka). À l'ouest, la grande cour du dairi était flanquée par deux édifices au nord le pavillon de la Bibliothèque, Kyōshoden 校書殿, et au sud le pavillon de la Tranquillité et du bonheur, Anpukuden 安福殿 - qui communiquaient par un passage couvert comportant en son centre une ouverture, la porte de l'Éclat de la lune, Gekkamon 月華門. Du côté de l'est, le ōniwa était bordé par un passage reliant deux pavillons - le pavillon du Bénéfique principe yō (ch. yang), Giyōden 宜陽殿, au nord et le pavillon des Plaisirs du printemps, Shunkyōden 春興殿, au sud - au milieu duquel s'ouvrait la porte de l'Éclat du soleil, Nikkamon 日華門. Le Kyūjōzu montre le tracé que 
suivait le petit canal mikawamizu dans cette partie de la résidence (pl. 9), tracé confirmé par le rouleau de peinture Nenjūgyōji emaki, qui indique même les passerelles en bois et les petits "ponts de pierre » ishibashi 石橋 que l'on empruntait pour le franchir. À l'angle nord-est du pavillon de la Bibliothèque, Kyōshoden, il y avait un espace réservé au tir à l'arc, le pavillon du Champ de tir à l'arc, Ibadono 弓場殿, qui est représenté en détail dans le quatrième rouleau du Nenjūgyōji emaki. À l'ouest du Kyōshoden et du Anpukuden, on trouvait quatre édifices dont le plus au nord était affecté à la Chancellerie privée, Kurōdo dokoro 蔵人所, et avait neuf travées de façade. À côté du Kurōdo dokoro s'élevait un petit pavillon, celui de l'office, Shinmotsu dokoro 進物所, qui était en charge de la vaisselle et de la présentation de la nourriture servie au souverain. L'angle sud-ouest de la résidence impériale était occupé par les deux bâtiments de l'atelier des Fabrications, Tsukumo dokoro 作物所, à qui incombait la réalisation d'objets et de travaux destinés à la cour. À l'est du Giyōden et du Shunkyōden, les deux édifices qui limitaient le côté est de la grande cour du dairi, il y avait deux petits édifices dont l'un abritait le palanquin impérial, Mikoshi yadori 御子宿, et l'autre le pavillon des Ustensiles rouges, Shukiden 朱器殿, où l'on rangeait la vaisselle cérémonielle en laque rouge.

19 Au nord du Shishinden s'élevait un bâtiment de même orientation appelé pavillon de la Bienveillance et du bonheur, Jijūden 仁寿殿, ou pavillon Arrière, Kōden 後殿 - par opposition au pavillon Avant que constituait le Shishinden - , où se déroulaient, entre autres, des banquets accompagnés de séances de composition poétique. Le Jijūden contenait aussi le magasin osamedono 納殿 de la Chancellerie privée, Kurōdo dokoro 蔵人 所, où l'on gardait l'encens réservé aux célébrations bouddhiques ainsi que l'or et l'argent destinés aux offrandes aux sanctuaires shintō. À l'est du Jijūden, il y avait une cour où poussaient deux pruniers et qui était limitée à l'est par un édifice de direction nord-sud, le pavillon des Brocarts, Ryōkiden 綾綺殿. À l'est du Ryōkiden et séparé de lui par deux petites cours, on trouvait un bâtiment orienté est-ouest, le pavillon de la Chaleur et de la clarté, Unmeiden 温明殿, où l'on conservait le miroir sacré. Le Unmeiden abritait par ailleurs le bureau du service intérieur du palais, Naishidokoro 内 待所.

\section{Le pavillon de Pureté et de Fraîcheur, Seiryōden}

L'édifice où l'empereur passait l'essentiel de ses journées se trouvait à l'ouest du pavillon de l'Étoile polaire, Shishinden, auquel il était relié par un passage nagahashi 長 橋. Ce bâtiment de onze travées du nord au sud sur cinq de l'est à l'ouest était appelé pavillon de Pureté et de Fraîcheur, Seiryōden 清凉殿 (pl. 7 et 8) ${ }^{31}$. Du côté de l'ouest, il était relié par trois passages orientés est ouest à un pavillon Arrière, Kōryōden ou Kōrōden 後涼殿, de neuf travées sur quatre avec lequel il formait une ensemble délimitant deux cours « en vase clos» tsubo 壸 appelées respectivement clos de la salle des Plateaux, Daibandokoro no tsubo 台盤所壳, et clos de la Salle à manger, Asagarei no tsubo 朝嗆㽦. D'après le ōkagami 大鏡 (Le Grand miroir, $\mathrm{XI}^{\mathrm{e}}$-XII ${ }^{\mathrm{e}}$ siècle), l'empereur Kazan (r. 984-986) aimait tant les chevaux qu'il aurait utilisé le corridor medō 馬道 qui divisait le Kōryōden en deux magasins osamedono 納殿 pour faire entrer une de ses montures favorites jusqu'au clos de la Salle à manger ${ }^{32}$. La façade orientale du Seiryōden était longée par une galerie de type mago hisashi doublée du côté extérieur par un plancher (sunoko 箐子). Elle s'ouvrait sur une vaste cour recouverte de sable et agrémentée de diverses espèces végétales où se déroulaient quantité de cérémonies et 
de rites en relation avec le cycle annuel des célébrations ${ }^{33}$. Le plan du dairi du Kyūjōzu indique clairement le tracé que suivait le petit canal mikawamizu qui passait devant cette partie de l'édifice ${ }^{34}$. Le mikawamizu est par ailleurs représenté à plusieurs reprises sur le Nenjūgyōji emaki. À en juger d'après ces documents, le canal pénétrait dans la résidence impériale à la hauteur de la porte nord-ouest, Shikikenmon, porte des Rites perpétuels, de l'enceinte extérieure du dairi, avant de franchir l'enceinte intérieure à proximité de la porte de la Clarté du principe In, Onmeimon 陰明門. Il passait ensuite le long de la façade nord du Kōryōden et du Seiryōden, c'est-à-dire tout près de la salle de bain de l'empereur. Tout au nord de la grande cour du Seiryōden, le mikawamizu prenait la forme d'une petite cascade takiguchi 瀧口 (la bouche du dragon). Il suivait ensuite les contours du pavillon de Pureté et de Fraîcheur et des édifices qui bordaient la façade ouest de la grande cour du sud de la résidence. Non loin de la porte de la Tranquillité éternelle, Eianmon 永安門, il prenait la direction de l'ouest jusqu'à l'angle sud-ouest de l'enceinte intérieure du dairi où il retrouvait la direction du sud et franchissait l'enceinte extérieure. Le parcours de ce canal était conforme aux règles de la géomancie qui voulait que l'eau circule de l'est (le Dragon Vert, seiry $\bar{u}$ 青龍) vers l'ouest (le Tigre Blanc, byakko 白虎) ou, mieux encore, vers le sud-ouest de façon à évacuer les énergies néfastes ${ }^{35}$.

Plan du pavillon de Pureté et de fraîcheur en français

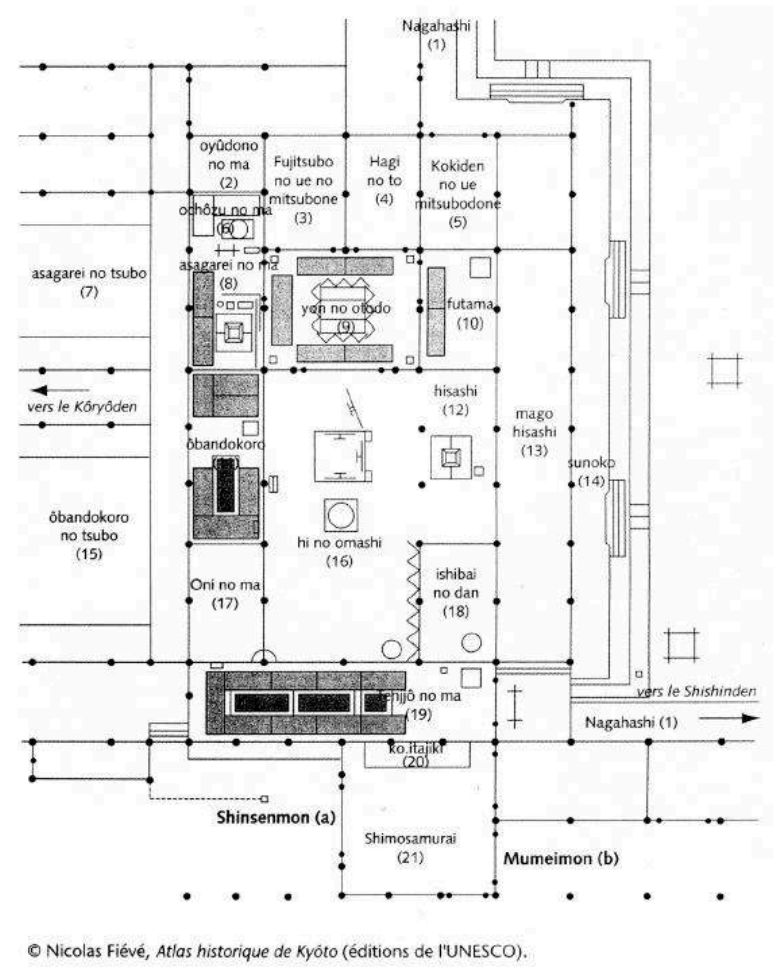

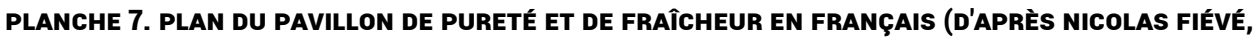
ATLAS HISTORIQUE DE KYōTO, UNESCO, 2008) 
(a) des immortels
(b) Sans Nom

cours et salles

(1) Long Pont

chambre réservete a la dame du Fujitsubo

* chamber des Lespedezes *

chambere réservée a la dame du Koliden

lieu ou rEmpereur se lawait les mains avant ses repas

cour

salle a manger de l'Empereur

Salle oú se trouvait la couche de PEmpereur

(10) salle de * deux intervalles *

(11) salle des plateaux

(12) gaierie

(13) seconde galerie

(13) seconde galerie

(14) galen

(16) salle où se trouvait rEmpereur pendant le jour

(17) chambre des peintures de demons

(18) leu des devotions de r'Empereur pour le sanctuaire d'ise

(19) salle d'en Haut

(20) petit plancher

(21) salle de service annexee

\section{PLANCHE 7 (SUITE). LÉgENDE}

Plan du pavillon de Pureté et de fraîcheur en japonais

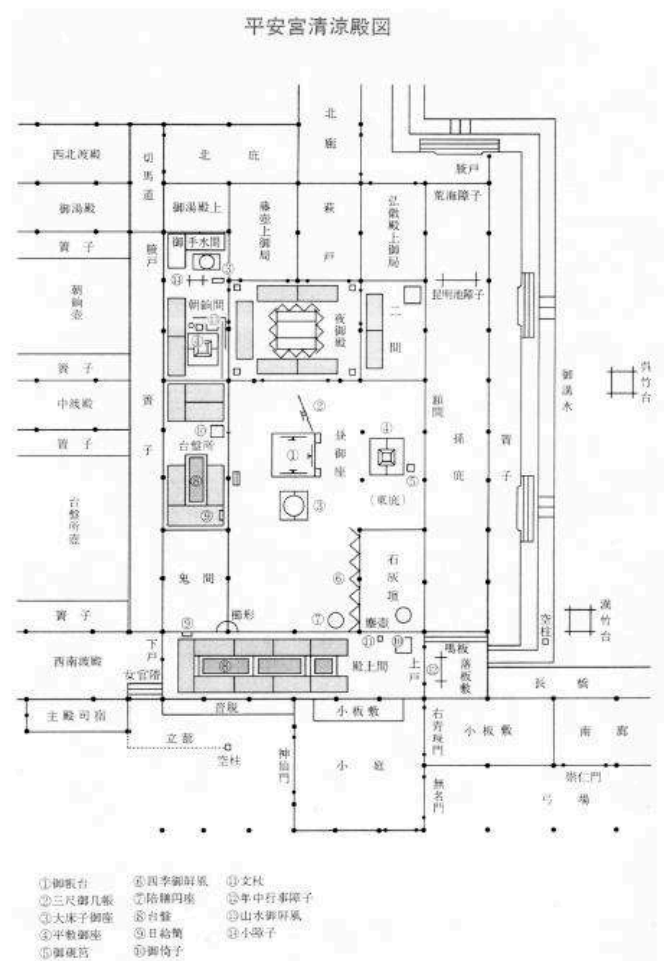

PLANCHE 8. PLAN DU PAVILLON DE PURETÉ ET DE FRAîCHEUR EN JAPONAIS (D'APRÈs NAgAHARA KEIJI ET AL., ÉD., NIHONSHI JITEN, IWANAMI SHOTEN, 1999, P. 1406)

21 L'organisation spatiale du Seiryōden était particulièrement complexe en raison du rôle exceptionnel du personnage qui y vivait et des nombreuses tâches qu'il devait 
accomplir. Comme dans la capitale des Tang, seule la partie nord du pavillon où résidait l'empereur avait un caractère privé. La partie méridionale du Seiryōden était pour l'essentiel réservée à l'accomplissement des fonctions officielles qui incombaient au souverain. Au sud, le Seiryōden était précédé par une sorte de vestibule constitué d'une salle, Shimozamurai 下侍, qui se prolongeait par une petite cour dotée de deux accès, la porte des Immortels, Shinsenmon 神仙門, à l'ouest, et la porte Sans Nom, Mumeimon 無名門, à l'est. De là, on accédait à un espace planchéié koitajiki 小板敷, ou " petit plancher ", qui conduisait à la salle d'En-Haut, Tenjō no ma 殿上間, où ne pénétraient que les nobles de cour (tenjōbito) dûment autorisés à le faire. À l'angle nord-est du Tenjō no ma, il y avait un siège réservé à l'empereur goishi 御椅子 à proximité duquel se trouvait une cloison mobile où figurait le calendrier des célébrations de la cour. L'appartement de jour du souverain, Hi no omashi ou Hi no goza 昼御座, occupait la partie centrale (moya) du Seiryōden. Du côté de l'est, il était bordé par une plate-forme dépourvue de plancher, Ishibai no dan 石灰壇, où le souverain célébrait chaque jour divers rituels en l'honneur du sanctuaire d'Ise. Du côté de l'ouest, il était limité par la chambre des démons, Oni no ma 鬼間, ainsi appelée en raison des peintures qu'elle contenait et par la salle des plateaux, Daibandokoro 台盤所, où attendaient les mets servis au souverain. Au nord de l'appartement de jour, il y avait la chambre à coucher de l'empereur, Yoru no otodo 夜御殿, dont les parois étaient recouvertes d'un enduit et pourvues de portes coulissantes. Si l'on en croit le Tsurezure gusa 徒然草 (Les Heures oisives, CXXXIII, XIV siècle) ${ }^{36}$, le chevet de l'empereur était orienté vers l'Est, de façon à ce que le souverain bénéficie de l'influence du principe $y \bar{o}$ (ch. yang). Pendant la nuit, le Yoru no otodo était éclairé par quatre lanternes, une à chaque angle de la pièce, qui dispensaient leur lumière jusqu'au lever du jour. C'est à cet endroit que se trouvait l'épée, un des trois regalia symbolisant le pouvoir de l'empereur. Plusieurs pièces jouxtaient le côté nord de l'appartement de nuit : la Salle à manger impériale, Asagarei no ma 朝飭間, la pièce aux ablutions préalables aux repas, Ochōzu no ma 御手水間, la salle de bain du souverain, Oyudono no ma 御湯殿間, la chambre de la dame du Fujitsubo, Fujitsubo no ue no mitsubone 藤萤上御局, la chambre aux Lespédèzes, Hagi no to 荻戸, ainsi appelée en raison des fleurs qui y étaient représentées, la chambre de la dame du Kokiden, Kokiden no ue no mitsubone 弘徽殿上御局, et pour finir, la salle de Deux travées, Futama 二間, qui contenait une image de Kannon. La résidence impériale n'abritait pas un seul établissement bouddhique digne de ce nom, en raison de la présence dans le dairi des regalia, symboles du pouvoir impérial. Les célébrations bouddhiques se déroulaient dans le Shingon.in fondé en 834 à l'instigation de Kūkai (774-835) et situé du côté ouest de l'enceinte extérieure de la résidence impériale. Le Shingon.in, appelé aussi Shūhōin 修法院, était entouré par une enceinte de pisé pourvue d'une porte sur chacun de ses côtés et il se composait de plusieurs bâtiments. Le Nenjūgyōji emaki donne une représentation détaillée de cet ensemble architectural ${ }^{37}$.

\section{Le palais Arrière, kōkyū}

22 Au nord de la partie de la résidence impériale réservée à l'empereur, le palais Arrière, $k \bar{k} k y \bar{u}$, formait un ensemble architectural constitué de sept pavillons den et de cinq clos sha où vivaient les épouses et les concubines du souverain ${ }^{38}$. Le centre du kōkyu était occupé par trois pavillons qui avaient des dimensions identiques, à savoir sept travées d'est en ouest sur deux du nord au sud, et la même orientation est-ouest. (pl. 5, 6 et 9). Le premier était le pavillon des Fragrances durables, Shōkyōden ou Jōkyōden 承香殿 ${ }^{39}$, 
qui s'élevait au nord du Jijūden et consistait en deux grandes salles séparées en leur milieu par un corridor medō 馬道. La tradition voudrait que le poète Ki no Tsurayuki 紀 貫之 ( ?- 945) ait compilé l'anthologie du Kokin wakashū (古今和歌集, Recueil de poèmes anciens et modernes) dans cet édifice. Le second pavillon, dit de la Tranquillité habituelle, Jōneiden 常寧殿 ${ }^{40}$, était parallèle au Shōkyōden et séparé de celui-ci par une vaste cour. Le troisième pavillon, celui de la Vision correcte, Jōganden 貞観殿 ${ }^{41}$, était affecté à la Garde-robe, Mikushigedono 御匣殿, qui était chargée des vêtements et des ornements impériaux, ainsi qu'au service de la maison de l'impératrice, Chūgūshiki 中 宮職. Du côté de l'ouest, les trois édifices qui se trouvaient au centre du palais Arrière étaient flanqués par deux bâtiments appelés pavillon de l'Expansion du beau, Kokiden 弘徽殿 ${ }^{22}$, et pavillon du Développement de l'éclat, Tōkaden 登花殿 ${ }^{43}$. Du côté de l'est, ils étaient bordés par deux autres pavillons, ceux de la Belle lumière, Reikeiden 麗景殿 ${ }^{44}$, et de l'Omniprésente lumière, Sen.yōden 宣輝殿 ${ }^{45}$. Exception faite du Shōkyōden, les pavillons du palais Arrière étaient tous reliés par un réseau de passages couverts qui formaient plusieurs cours intérieures, entre autres celle du puits du quartier de l'impératrice, kisaimachi no $i$ 后町井, qui est mentionné dans une anecdote du Konjaku monogatari (Histoires qui sont maintenant du passé, livre XXIV, anecdote 6) ${ }^{46}$, et sur le plan du Kyūjōzu (pl. 9).

Le palais Arrière contenait par ailleurs cinq clos sha 舍 ${ }^{47}$ qui auraient été construits en 842 (Jōwa, 9), du temps de l'empereur Ninmyō (仁明天皇, r. 833-850), c'est-à-dire près d'un demi-siècle après la fondation de la capitale. Ces édifices étaient tous orientés nord-sud et ils présentaient la particularité d'avoir deux noms, un nom chinois - à connotation fortement taoïste comme ceux des autres bâtiments du palais et de la résidence impériale - et un nom japonais composé du mot tsubo 盇 et du nom d'une des espèces végétales qui poussaient dans le jardin sur lequel ils donnaient. Tsubo, au sens premier du terme, c'est une grande jarre, un espace clos, qui dans le cadre de la résidence impériale désigne une petite cour couverte de sable ou de gravier, un minuscule jardin où poussent un ou quelques végétaux. À l'époque d'Edo, le terme tsubo s'appliquera aux minuscules jardins intérieurs que l'on trouve dans les maisons des marchands machiya 町家 de cette période. Les clos du palais Arrière avaient tous cinq travées d'est en ouest sur deux du nord au sud et c'est là que résidaient les épouses et les concubines de l'empereur. Ils formaient deux ensembles architecturaux situés de part et d'autre des sept pavillons qui occupaient le centre du palais Arrière. À l'ouest, le clos des Effluves parfumés, Higyōsha 飛香舎, appelé aussi clos de la Glycine, Fujitsubo 藤盇, se trouvait à proximité du Seiryōden. Il était relié par deux passages au clos des Fleurs non gélives, Gyōkasha 凝花舎 - que l'on désignait aussi sous le nom de clos du Prunier, Umetsubo 梅盇 - avec lequel il formait une cour. L'angle nord-ouest du dairi était occupé par un troisième clos, celui des Senteurs multiples, Shūhōsha 襲芳舎, appelé aussi clos du Tonnerre, Kaminari no tsubo 雷鳴㱬, parce qu'il avait été frappé par la foudre. Du côté de l'est, il y avait deux clos - le clos du Radieux principe yō, Shōyōsha 昭陽舎, appelé aussi clos du Poirier, Nashitsubo 梨盇, et le clos du Paysage frais comme le printemps, Shigeisha 淑景舎, ou clos du Paulownia, Kiritsubo 桐盇 - qui présentaient chacun la particularité d'être doté d'un pavillon annexe au nord. Le clos du Poirier et le clos du Paulownia se composaient chacun de deux bâtiments situés dans le prolongement l'un de l'autre et reliés entre eux par des passages. Les deux tsubo de l'est comme les trois de l'ouest étaient reliés entre eux et à certains bâtiments par divers passages et autres couloirs, tout en étant isolés par des enceintes dotées de petites portes (pl. 9). Si le nom des clos du palais Arrière faisait référence à des plantes, 
il se peut que ce soit aussi parce que dans les capitales chinoises, c'est dans la partie la plus septentrionale du palais que se trouvaient les jardins impériaux où poussaient, entre autres, des espèces végétales célébrées par les poètes ${ }^{48}$.

\section{Le clos du Paulownia, Kiritsubo}

24 Le clos du paulownia, Kiritsubo (ou Shigeisha, clos du Paysage frais comme le printemps), se trouvait à l'extrémité nord-est du palais Arrière et de l'univers rigoureusement compartimenté et tourné vers l'intérieur que constituait la résidence impériale (pl. 5 et 6). Le plan du Kyūjōzu montre de façon particulièrement éloquente la longueur et la complexité du trajet que Kiritsubo, l'héroïne du premier chapitre du Roman du Genji, devait parcourir pour accéder au Seiryōden, le pavillon où résidait le souverain (pl. 9). Murasaki Shikibu précise par la suite que c'est au Kiritsubo que se trouvaient les appartements du prince Genji au palais, alors même que quelques lignes plus haut, elle rapporte que celui-ci, alors âgé de douze ans vient de célébrer le genpuku 元服, c'est-à-dire le rituel du passage à l'âge adulte. Il semble pour le moins surprenant que le prince loge dans le palais Arrière, celui-ci étant en principe exclusivement réservé aux épouses impériales, aux concubines et éventuellement aux enfants en bas âge qu'elles avaient de l'empereur. Qui plus est, il y avait dans l'enceinte même de la résidence impériale des emplacements prévus pour l'hébergement des ministres entre autres au nord du Giyōden, pavillon du bénéfique Principe yō, où le grand chancelier passait la nuit. Sans doute Murasaki Shikibu a-t-elle voulu ainsi montrer l'attachement extrême que portait l'empereur à ce fils né d'une femme qu'il avait si passionnément aimée et à cause de laquelle il avait déjà franchi les limites de ce qu'il pouvait se permettre. Murasaki Shikibu n'hésite d'ailleurs pas à qualifier le souverain qui apparaît dans le premier chapitre de son roman - sans doute inspiré par Daigo tennō 醍醐天皇 (r. 885-930) ${ }^{49}$ - sous le nom de Kiritsubo no mikado 桐盇帝, le souverain du clos du Paulownia. Dans le chapitre XXXII du Roman du Genji qui a pour titre Umegae 梅枝 (La branche de prunier), le prince Genji installe sa fille Akashi no nyōgo 明石女御 ${ }^{50} \mathrm{M}$ VB2012-02-27T18:53:00dans son ancienne résidence du clos du Paulownia. D’après le Ōkagami, Fujiwara no Genshi 藤原原子 (ou Motoko,?-1002), la seconde fille de Fujiwara no Michitaka 藤原道隆 (953-995), aurait résidé au Shigeisha lorsqu'elle est devenue l'épouse du prince impérial (le futur empereur Sanjō 三条天皇 r. 1011-1016). De ce fait, on l'aurait aussi appelée Shigeisa (variante de Shigeisha) ${ }^{51}$, conformément à l'usage qui voulait que l'on désigne les dames de la cour par le nom de l'édifice où elles vivaient. Murasaki Shikibu a peut-être choisi de faire résider au Kiritsubo la malheureuse favorite de l'empereur parce qu'elle se souvenait de la fin tragique de Fujiwara no Genshi, sans doute victime d'une rivale. Par ailleurs, à en juger par le rang élevé des personnages qui, selon les sources, ont résidé au Kiritsubo, le fait que ce pavillon fût éloigné du Seiryōden ne signifie nullement que ses occupants étaient moins bien considérés que ceux qui vivaient plus près du souverain. Le « clos du paulownia " était situé dans la partie est de la résidence impériale, ce qui revient à dire, d'après le système d'interprétation du monde propre au taoïsme, qu'il était associé au principe $y \bar{o}$ (yang) et au printemps, comme l'indique d'ailleurs son nom chinois.

Kiri (paulownia tomentosa Steudel), le paulownia, auquel le premier chapitre du Genji monogatari doit son titre, est un arbre d'origine chinoise à feuilles caduques et aux grandes fleurs mauves campanulées qui a été introduit et s'est développé très tôt au 
Japon. Il fait partie de la famille des scrofulariacées (goma no hagusa) et on le désigne aussi sous le nom de hakutō 白桐 (littéralement « paulownia blanc ») ${ }^{52}$.

Si l'on en juge par la suite du Roman du Genji, la couleur violette (murasaki) de la fleur de paulownia, comme de la glycine d'ailleurs, était loin d'être indifférente à son auteur, d'autant qu'elle correspondait à l'échelon le plus élevé dans la hiérarchie non seulement des couleurs, mais aussi de l'administration. Les costumes de cour des fonctionnaires du premier rang étaient en effet violet vif et ceux des second et troisième rangs, violet pâle. Dans la classification des couleurs définie par la théorie chinoise des cinq agents (wuxingshuo 五行説, jap. gogyōsetsu), la couleur mauve est associée au centre et à l'empereur ${ }^{53}$.

Mais en dépit de la place de choix que Murasaki Shikibu lui a donné dans son œuvre, le paulownia est infiniment moins présent que d'autres espèces végétales dans les sources écrites, ne serait-ce qu'en comparaison de la glycine fuji 藤 (Wistaria floribunda D. C.) qui est déjà mentionnée dans le Kojiki 古事記. Celle-ci apparaît en effet près de trente fois dans le Man.yōshū et encore plus souvent dans les anthologies poétiques ultérieures où les poètes évoquent volontiers l'image de la glycine qui s'enroule autour du pin.

Le paulownia ne figure pas parmi les plantes citées dans les Règlements de l'ère Engi, pas plus dans le chapitre de l'office des Plantes médicinales Ten.yakuryō 典薬寮 ${ }^{54}$ que dans ceux de l'office des Constructions Mokuryō 木工寮 ${ }^{55}$ ou de l'office des Fabrications Naishōryō 内匠㡍 ${ }^{56}$. Étant donné le nombre considérable d'objets dont on sait qu'ils étaient fabriqués avec du bois de paulownia, on est en droit de s'étonner qu'il ne se trouve pas dans cet ouvrage. Il est vrai que, d'une manière générale, le Engishiki ne donne pas le nom des essences utilisées dans la fabrication d'objets. Quoi qu'il en soit, le terme kiri y est seulement mentionné à propos de deux sanctuaires appelés respectivement Kiri no jinja 桐神社, sanctuaire des Paulownias, et Kirihara jinja 桐原神 社, sanctuaire de la Plaine des paulownias.

Le paulownia est cependant évoqué dans les poèmes 809 et 810 du Man.yōshū, où Ōtomo no Tabito 大伴旅人 (665-731) fait allusion à une cithare du Yamato taillée dans un paulownia du mont Yuhishi, qui se trouve dans l'île de Tsushima, et dont les arbres avaient la réputation de donner des instruments incomparables. Le poète rapporte comment la cithare lui est apparue en rêve sous les traits d'une jeune fille. Celle-ci lui explique qu'elle a eu la chance de rencontrer un habile artisan qui l'a abattue pour en faire une cithare à six cordes (wagon ou yamatogoto 倭琴). La jeune fille/cithare compose le poème suivant :

\begin{tabular}{|l|l|}
\hline Ikani aramu & Ah ! que vienne le jour \\
\hline Hi no toki ni ka mo & que vienne l'heure \\
\hline Koe shiramu & où sur les genoux \\
\hline Hito no hiza no we & d'un homme qui connaît l'art des sons \\
\hline Waga makura kamu & je poserai ma tête. \\
\hline
\end{tabular}

いかにあらむ日の時にかも音知らむ人の榺の上我が枕かむ

Et Ōtomo no Tabito de répondre : 


\begin{tabular}{|l|l|}
\hline Koto tohanu & Cithare, quoique faite \\
\hline Ki ni ha aritomo & d'un bois dépourvu de voix \\
\hline Uruhashiki & vous êtes à même \\
\hline Kimi ga tanare no & de recevoir les faveurs \\
\hline Koto ni shi arubeshi & d'un seigneur magnifique ${ }^{57}$. \\
\hline
\end{tabular}

\section{言問はぬ木にはありともうるはしき君が手馴れの琴にしあるべし}

Mis à part les deux pièces du Man.yōshū mentionnées plus haut, il existe une quinzaine d'autres poèmes japonais (waka) où il est question du paulownia. Le Shinkokin wakashū (Nouvelle anthologie de poèmes anciens et modernes ${ }^{58}$ ) en contient un (automne, ge, 534). Dans le Fuboku wakashō (夫木和歌抄 Choix de poèmes japonais ${ }^{59}$ ), il y en a cinq ${ }^{60}$, dont quatre figurent dans la section «Paulownia » du volume 15, consacré à l'automne, entre autres le poème suivant du moine Jakuren 寂蓮 :

\begin{tabular}{|l|l|}
\hline Momoshiki ya & Le phénix réside \\
\hline Kiri no kozuwe ni & au sommet du paulownia, \\
\hline Sumu tori no & au palais impérial, \\
\hline Chitose ha take no & sans que change jamais la couleur des bambous \\
\hline Iro mo kaharazi & mille ans durant. \\
\hline
\end{tabular}

\section{ももしきやきりの木ず总すむ鳥のちとせは竹の色もかはらじ 61}

31 Dans le volume 21 du Fuboku wakashō, consacré à des poèmes divers, on trouve par ailleurs un poème où Fujiwara no Shunzei 藤原俊成 associe lui aussi la cithare et le paulownia :

\begin{tabular}{|l|l|}
\hline Suzukayama & À traverser \\
\hline Kiri no furuki no & le vieux pont en rondins de paulownia \\
\hline Marokibashi & de la barrière de Suzuka, \\
\hline Kore mo ya koto no & on croirait entendre \\
\hline Ne ni kayofuran & résonner une cithare. \\
\hline
\end{tabular}

すずか山きりのふるきのまろきばしこれもやことのねにかよふらん62

La vingt-et-unième et la dernière anthologie impériale de waka, Shinshoku kokin wakashū (Suite du nouveau recueil de poèmes anciens et modernes), qui date de 1439, contient 
elle aussi un poème dans lequel Sanjōnishi Kinyasu 三条西公保 (?-1460) évoque le paulownia:

\begin{tabular}{|l|l|}
\hline Kage takaki & La voix du phénix \\
\hline Kiri no kozuwe ni & qui réside au sommet \\
\hline Sumu tori no & du paulownia altier \\
\hline Kowe machi iden & augure de la majesté \\
\hline Miyo no kashikosa & du nouveau règne. \\
\hline
\end{tabular}

かげ高き桐の木末にすむ鳥のこ急待ちいでん御代のかしこさ63

Sei Shōnagon écrit, quant à elle, ce qui suit à propos du paulownia, dans la section «Fleurs des arbres » du Makura no sōshi (Notes de chevet ${ }^{64}$ ):

La fleur violet-pourpre du paulownia est aussi très jolie. Je n'aime pas la forme de ses larges feuilles étalées; cependant, je n'en puis parler comme je ferais d'un autre arbre. Quand je pense que c'est dans celui-ci qu'habiterait l'oiseau fameux en Chine [le phénix], je ressens une impression singulière.

À plus forte raison, lorsque avec son bois on a fabriqué une cithare et qu'on en tire toutes sortes de jolis sons, les mots ordinaires suffisent-ils pour vanter le charme du paulownia? C'est un arbre vraiment superbe ${ }^{65}$.

34 À en juger par les poèmes qui précèdent et le passage que Sei Shōnagon consacre à cet arbre, le paulownia semble étroitement lié au pouvoir impérial et au phénix, symbole par excellence de la pérennité de ce pouvoir. Il n'y a donc rien d'étonnant à ce que Murasaki Shikibu ait choisi d'en faire le titre du premier chapitre de son roman et le nom de la première héroïne de son œuvre. Mais le paulownia semble aussi entretenir des rapports très étroits avec la musique et en particulier le son de la cithare qui apparaît à maintes reprises dans la trame du Roman $d u$ Genjii ${ }^{66}$ où il ponctue les émotions et les passions des héros.

Dans le premier chapitre de son roman, Murasaki Shikibu, ne donne guère d'indications précises sur le cadre où se déroule l'action, comme c'est d'ailleurs souvent le cas dans les œuvres de ce type. Si l'on ne disposait pas de textes et de documents iconographiques de l'époque, il serait fort difficile de se faire une idée du contexte matériel dans lequel évoluent les nombreux personnages du monde de la cour de Heian qu'elle met en scène. Mais le Genji monogatari n'en donne pas moins un éclairage intéressant sur le rôle considérable que jouait dans la vie de la cour le système de correspondances entre la nature, l'homme et la société, propre au taoïsme. Murasaki Shikibu semble avoir été elle-même très influencée par la vision symbolique du monde qui a présidé à la construction du palais où elle nous entraîne à la suite de ses personnages. 


\section{NOTES}

1. Voir Francine Hérail, «Les incendies autour du palais impérial de 960 à 1068 », in Nicolas Fiévé (éd.), Atlas historique de Kyoto, UNESCO, 2008.

2. Oboroya Hisashi, « Heiankyō-Ōchō no fūkei », in Heian kizoku no kankyō, Shibundō, 1994, p. 67, avec une carte établie d'après celle du Seikaigansho (清懈眼抄, Recueil des observations du bureau de police), une compilation de la fin du XII ${ }^{\mathrm{e}}$ siècle établie par un membre de la famille Kiyohara.

3. À ce sujet voir Kinda Akihiro, Kochizu kara mita kodai Nihon, Tōkyō, coll. "Chūkō shinsho", Chūō kōronsha, 1999.

4. Kyūjō zu, coll. «Yōmei sōsho : Kiroku monjo », Besshū, Shibunkaku shuppan, 1996.

5. Engishiki, coll. «Shintei zōho kokushi taikei », livre III, p. 919-930. On notera que les plans Kyūzu ne figurent pas dans cette édition.

6. Daidairizu kōshō, coll. « Zōtei kojitsu sōsho », 3 vols.

7. Nenjūgyōji emaki, coll. « Nihon no emaki (Konpakuto ban)», vol. 8.

8. Voir Marc Kalinowski, Cosmologie et divination dans la Chine ancienne - Le compendium des cinq agents (Wuxing dayi), Paris, EFEO, 1991, p. 57-74. Marcel Granet, La Pensée chinoise, Paris, Albin Michel, 1934, p. 77-91. Michèle Pirazzoli-t'Serstevens, Architecture universelle: Chine, Fribourg, Office du Livre, 1970, pp. 10-12.

9. Michèle Pirazzoli-t'Serstevens, op. cit., pp. 44-46.

10. Au sujet de la ville de Heian, voir Daidairizu kōshō, op. cit., vol. 1, pp. 1-31. Oboroya Hisashi, in Heian kizoku no kankyō, op. cit., pp. 28-75. Nicolas Fiévé, L'Architecture et la ville du Japon ancien, Paris, Maisonneuve et Larose, 1996; et pour une description détaillée, Marie Maurin, "L'organisation spatiale de la capitale », in Atlas historique de Kyoto, op. cit.

11. D'après Tsuji Jun.ichi, qui fait partie du Kyōto-shi maizō bunkazai kenkyūjo, dans le cas de Heiankyō, 1 jō équivaut à 2,98445 mètres (communication personnelle). Voir Tsuji Jun.ichi, « Heiankyō no kibo », Kikan kōkogaku 49, Kyōto, octobre 1994.

12. À propos du daidairi, voir Daidairizu kōshō, op. cit., vol. 1, p. 32-316. Satō Makoto, «Daidairi » in Heian kizoku no kankyō, Shibundō, 1995, p. 76-92. Francine Hérail, Notes journalières de Fujiwara no Michinaga, traduction du Midō kanpakuki, Droz, vol. 1, 1987, pp. 113-125.

13. Voir Michèle Pirazzoli-t'Serstevens, op. cit., pp. 44-46 et 96.

14. Shigisan engi emaki, coll. « Nihon no emaki (Konpakuto-ban)», op. cit., vol. 4, pp. 39-41.

15. Francine Hérail, Notes journalières de Fujiwara no Sukefusa, traduction du Shunki, Droz, vol. 2, 2004, pp. 180-195.

16. Pour une description détaillée du Hasshōin et du Burakuin, voir Marie Maurin, «L'organisation spatiale du palais », op. cit.

17. Au sujet de la résidence du prince impérial, voir Daidairizu kōshō, op. cit., vol. 3, pp. 323-334, et en particulier le plan de la page 331.

18. Nihon Montoku tennō jitsuroku, Kajō, $3^{\mathrm{e}}$ année, $3^{\mathrm{e}}$ lune, $21^{\mathrm{e}}$ jour.

19. Shōyūki, Chōgen, $4^{\mathrm{e}}$ année, $8^{\mathrm{e}}$ lune, $25^{\mathrm{e}}$ jour (1031).

20. À propos du Butokuden, voir Engishiki, op.cit., vol.2, livre XVII (Naishōryō), pp. 447-464. Daidairizu kōshō, op. cit., vol. 1, p. 305-314 ; vol. 3, pp. 449-450.

21. Shinsarugakuki, coll. « Tōyō bunko », vol. 424, Heibonsha, 1983, pp. 117-120.

22. Voir Tsuji Jun.ichi, "Heiankyō no tatemono ", in Kikan kōkogaku 49, Kyōto. Nicolas Fiévé, «L'architecture noble à l'époque de Heian (784-1185) », in Atlas historique de Kyōto, op. cit.

23. À ce sujet voir Nenjūgyōji emaki, op. cit., p. 35-36; Ban dainagon emaki, coll. Nihon no emaki (Konpakuto ban, op. cit., vol.2, pp.12, 13, 41, 42. Akiyama Ken, Komachiya Teruhiko, Sugai Minoru (éd), Genji monogatari zuten, Tōkyō, Shōgakukan, 1997, pp. 12-21. 
24. Dans l'architecture traditionnelle japonaise, la longueur du ken équivaut à six pieds shaku, soit environ 1,80 mètre. Mais à l'époque de Heian, il en allait tout autrement. Voir Tsunoda Bun.ei et al. (éd.), Heian jidaishi jiten, Kadokawa shoten, 1994, p. 2340 (ma).

25. À ce sujet voir Tsunoda Bun.ei et al. (éd.), Heian jidaishi jiten, Kadokawa shoten, 1994, pp. 798 (kenmen shihō).

26. Au sujet du Yingzao fazi voir Chen Mingda, Yingzao fashi damuzuo yanjin, Beijing, Wenwu Press, 1981. Paul Demiéville, Ying tsa fa che, BEFEO, 1925.

27. Au sujet de la résidence impériale dairi, voir Daidairizu kōshō, op. cit., vol. 1, p. 317-428, vol. 2, p. 1-436. Kimura Shigemitsu, Heiankyō kurashi to fūkei, Tōkyōdō shuppan, Tōkyō, 1994, pp. 37-47. Marie Maurin, « L'organisation spatiale du palais », op. cit.

28. Tsunoda Bun.ei et al. (éd.), Heian jidaishi jiten, Kadokawa shoten, 1994, p. 1275.

29. Nenjūgyōji emaki, op. cit., pp. 31-32.

30. Voir Michèle Pirazzoli-t'Serstevens, op. cit., pp. 96-97.

31. Au sujet du Seiryōden et du Kōryōden, voir Daidairizu kōshō, op. cit., vol. 2, p. 68-234, et vol. 3, p. 455-456. Pour un plan détaillé, voir Genji monogatarizuten, op. cit, p. 19.

32. Ōkagami, NKBT, vol. 21, pp. 145-147.

33. Voir Nenjūgyōji emaki, op.cit., p. 34 et suiv. Marie Maurin, «De l'espace primordial au jardin d'agrément », in Éloge des sources, pp. 294-295.

34. Au sujet du mikawamizu, voir aussi Daidairizu kōshō, op. cit., vol. 2, pp. 94-97.

35. Voir Marc Kalinowski, op. cit., pp. 57-62.

36. Tsurezuregusa, NKBT, vol. 30, p. 196.

37. Voir Heian jidaishi jiten, op. cit., p. 1285. Nenjūgyōji emaki, op. cit., p. 33.

38. Au sujet du palais Arrière, voir Hinata Kazumasa, « Dairi . kōkyū », in Heian kizoku no kankyō, op. cit., pp. 93-132.

39. Au sujet du Shōkyōden, voir Daidairizu kōshō, op. cit., vol. 2, pp. 289-299.

40. Ibid., vol. 2, pp. 402-413.

41. Ibid., vol. 2, pp. 414-417.

42. Ibid., vol. 2, pp. 417-425.

43. Ibid., vol. 2 , pp. 426-430.

44. Ibid., vol. 2, pp. 431-433.

45. Ibid., vol. 2, pp. 433-436.

46. Konjaku monogatari shū, NKBT, vol. 25, p. 283.

47. Au sujet des cinq clos, gosha, voir Daidairizu kōshō, op. cit., vol. 3, pp. 1-33.

48. À ce sujet, voir Hiraoka Takeo et Imai Kiyoshi, Tōdai no chōan to rakuyō shiryōhen, Kyōto daigaku, Kyōto, 1956. Song Minqiu (1019-1079), Chang'an zhi, éd. Siku quanshu, Taipei, 1931. Michèle Pirazzoli-t'Serstevens, op. cit., pp. 95-97.

49. Voir dans ce volume l'article de Takada Hirohiko, p. 62, note 25.

50. Genji monogatari, op. cit., vol. 16, p. 169.

51. Ōkagami, NKBT, tome 21, p. 179. Je remercie Francine Hérail pour cette précieuse information. D'après le Eiga monogatari, Fujiwara no Genshi serait morte subitement en 1002, après que « du sang lui fut sorti du nez et de la bouche ». À en croire des rumeurs de l'époque, il pourrait s'agir d'un assassinat commandité par une épouse impériale. Eiga mononogatari, tome 1, NKBT, vol. 75, 1964, pp. 234-235.

52. Voir Makino Tomitarō, Shin Nihon-shokubutsu-zukan, Hokuryūkan, 1961, p. 554.

53. Voir Marc Kalinowski, Cosmologie et divination dans la Chine ancienne - Le compendium des cinq agents (Wuxing dayi), Paris, EFEO, 1991, pp. 44-45.

54. Engishiki, livre XXXVII, op. cit., vol. 3, pp. 815-844.

55. Engishiki, livre XXXIV, ibid., vol. 3, pp. 783-796.

56. Engishiki, livre XVII, ibid., vol. 2, pp. 447-464. 
57. Man.yōshū, tome 1, Livre V, poèmes 810-811, coll. SNKBT, vol. 1, 1999, p. 459-460 ; traduction de l'auteur ; voir aussi René Sieffert, Man.yōshū Livres IV à VI, Paris, POF, UNESCO, pp. 183-185.

58. Le Shinkokin wakashū, qui date du début du XIII ${ }^{\mathrm{e}}$ siècle, est la huitième anthologie compilée sur ordre impérial.

59. Le Fuboku wakashō est une anthologie thématique monumentale qui a été compilée au début $\mathrm{du} \mathrm{XIV}^{\mathrm{e}}$ siècle et contient près de dix-huit mille poèmes.

60. Voir Hirata Shinobu, Waka shokubutsu hyōgen jiten, Tōkyōdō shuppan, Tōkyō, 1994, p. 93-94. Les autres pièces du volume $15 \mathrm{du}$ Fuboku wakashō où il est question du paulownia sont les poèmes 6086 de Fujiwara no Ietada, 6087 de Fujiwara no Tameie, et 6088 de Minamoto no Michitomo.

61. Fuboku wakashō, poème 6085 , in coll. "Shinpen kokka taikan », tome 2, Shisenshū-hen, Kadokawa shoten, 1984, p. 598,

62. Ibid. Section « Pont en rondins ", poème 9387, p. 671.

63. Livre 7, Poèmes de célébration, poème 789, ibid., tome 1, Chokusenshū hen, 1983, p. 738.

64. Makura no sōshi, NKBT, vol. 19, chap. 37, p. 84.

65. Notes de chevet, trad. André Beaujard, coll. «Connaissance de l'Orient », UNESCO, 1966, p. 63.

66. À propos du paulownia et du Roman du Genji, voir Hiroe Minosuke, «Genji monogatari no shokubutsu », in Koten shokubutsu zensh̄̄, vol. 5, Ariake shobō, Tōkyō, 1969, pp. 133-135.

\section{RÉSUMÉS}

Murasaki ne donne que peu d'information sur le palais impérial. C'est par le biais de sources écrites et iconographiques, certaines datant de l'époque de Heian, qu'il est possible de s'en faire une idée. Les valeurs symboliques du paulownia sont également étudiées.

Because Murasaki provides little information on the Imperial palace, we can only gain an idea of it through written and iconographic sources. The symbolic value of Paulownia is also studied.

\section{INDEX}

Index chronologique : Heian

キーワード : shuto 首都, daidairi 大内裏, dairi 内裏, Genji monogatari 源氏物語, Kiritsubo 桐㑒, Murasaki Shikibu 紫式部 (v. 973-v. 1014 ou 1025), kiri 桐, Heiankyō 平安京, Kyōto 京都, Heian jidai 平安時代 (794-1185), kenchikugaku 建築学

Thèmes : architecture

Index géographique : Heiankyō, Kyōto

Mots-clés : capitale impériale, Murasaki Shikibu (v. 973-v. 1014 ou 1025), dairi, Dit du Genji, Genji monogatari, daidairi, palais impérial, paulownia

Keywords : Architecture, Murasaki Shikibu (v. 973-v. 1014 or 1025), daidairi, dairi, Genji monogatari, Heiankyō, Kiritsubo, Kyōto, Tale of Genji 